\title{
Even Moderate Visual Stimuli Leads to Behavioural Responses in Term and Preterm Infants
}

\author{
Zores $C^{a, b}$, Vincent Va, Marchal $A^{a}$, Davy M ${ }^{a}$, Astruc $D^{a}$, Dufour $A^{b}$, Kuhn Pa,c \\ aédecine et Réanimation du nouveau-né, Service de Pédiatrie 2, Pôle Médico-Chirurgical Pédiatrique, Hôpital de Hautepierre, \\ Centre Hospitalier Universitaire de Strasbourg, Strasbourg, France.

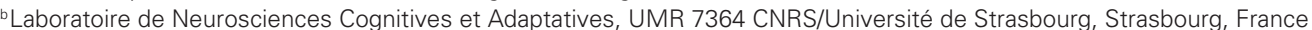 \\ ${ }^{c}$ Institut des neurosciences cellulaires et intégratives, Déterminants moléculaires de la douleur Strasbourg, France
}

\section{Introduction}

Very preterm infants (VPI) are exposed to atypical visual stimuli in the hospital. Their visual system was shown to be mature enough to allow them to physiologically react to ecological visual stimuli in the NICU from 28 weeks post-menstrual age (PMA). ${ }^{1}$ These stimuli have also been shown to induce sleep disruption. ${ }^{2}$ However, little is known about their behavioural responses to light level changes of different amplitudes, encountered in the hospital environment.

\section{Aims}

We aimed to evaluate the behavioural responses of VPIs to light level changes of different intensities. Our goal was also to follow longitudinally the development of these responses in VPIs until term corrected age and to compare them to the responses of fullterm newborn infants.

\section{Methods}

We included 25 term newborn infants and 26 VPIs at Strasbourg University Hospital (France) from September 2016 to June 2017. The VPIs were studied at three distinct PMA: 32, 36 and 40 weeks PMA. Infants were recorded during sleep when they were lying on their back, in a nest. Three different light stimulations (34, 100 and 300 lux above basal light level) were presented in a random order for a duration of 5 seconds. Video recordings were centred on the infant's face to appreciate their behavioural responses assessed by the NFCS (Neonatal Facial Coding System) score, reduced to four items, in 10 seconds epochs. ${ }^{3}$ The maximum NFCS score over the period of 60 seconds post stimulation was retained and was compared to the NFCS score determined during the 10 seconds preceding the stimulation ( $\mathrm{T}$ test).

\section{Results/Findings}

We observed a significant increase in the mean NFCS scores from the pre-stimulation period to the post-stimulation period, represented by the mean of maximal scores of post stimulation periods. This was noted regardless of the intensity of stimulation and whatever the PMA (all, $\mathrm{p}<0.05$ ). In preterm infants at 40 weeks PMA, means NFCS score were significantly higher at $100 \operatorname{lux}\left(\mathrm{T}_{(36)}=2.9, \mathrm{p}=0.006\right)$ and at $300 \operatorname{lux}\left(\mathrm{T}_{(36)}=2.8\right.$, $\mathrm{p}=0.009)$ compared with those following 34 lux stimulations: respectively $1.6(+/-1.0)$ and $1.6(+/-1.1)$ vs $0.72(+/-0.83)$ for 34 lux. We found this same difference at 32 weeks of PMA. The average NFCS score to 34 lux stimuli was $1.2+/-0.9$ versus 1.8

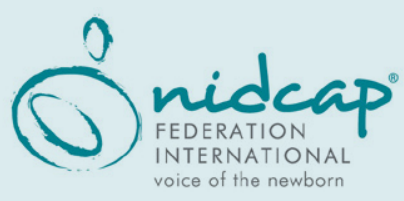

\section{Mission}

The NFI promotes the advancement of the philosophy and science of NIDCAP care and assures the quality of NIDCAP education, training and certification for professionals and hospital systems.

Adopted by the NFI Board, April 29, 2017

\section{Vision}

The NFI envisions a global society in which all hospitalized newborns and their families receive care in the evidence-based NIDCAP model. NIDCAP supports development, enhances strengths and minimizes stress for infants, family and staff who care for them. It is individualized and uses a relationship-based, family-integrated approach that yields measurable outcomes. 
$+/-0.9$ at $100 \operatorname{lux}\left(\mathrm{T}_{(36)}=2.3, \mathrm{p}=0.02\right)$ or $1.9+/-0.9$ to $300 \operatorname{lux}$ $\left(\mathrm{T}_{(37)}=2.3, \mathrm{p}=0.02\right)$. The mean NFCS score was significantly higher at 32 compared to 36 weeks PMA for a 100 lux light stimulation: $1.9(+/-0.8)$ vs $\left.1(+/-1.1)\left(\mathrm{T}_{(40)}=2.8, \mathrm{p}=0.04\right)\right)$. Similar results were observed for a 300 lux light stimulation: 1.9 $(+/-0.9)$ versus $1.2(+/-1.2)\left(\mathrm{T}_{(42)}=2.0 ; \mathrm{p}=0.05\right)$. The main results are summarized in Figure 1.

\section{Conclusion}

Even low-intensity visual stimulation leads to behavioural responses, measurable through scales evaluating pain and discomfort. This was present in all subgroups of infants, regardless of the level of immaturity of the newborn infants. The level of light stimulation and gestational age appeared to be the main determi- nants of the intensity of discomfort. All these results have clinical implications and support the need for better attention to light level changes during the hospital stay. ${ }^{4}$

References

1. Zores C, Dufour A, Pebayle T, Langlet C, Astruc D, Kuhn P. Very preterm infants can detect small variations in light levels in incubators. Acta Paediatrics 2015;104:1005-11.

2. Zore, C, Dufour A, Pebayle T, Dahan I, Astruc D, Kuhn P. Observational study found that even small variations in light can wake up very preterm infants in a neonatal intensive care unit. Acta Paediatrics 2018;107:1191-97.

3. Grunau RE, Oberlander T, Holsti L, Whitfield MF. Bedside application of the Neonatal Facial Coding System in pain assessment of premature neonates. Pain 1998;76: 277-86.

4. White RD. Defining the optimal sensory environment in the NICU: an elusive task. Acta Paediatrics 2018;107: 1112-1112.

\section{FIGURE 1. Variation in NFCS score by population and intensity}

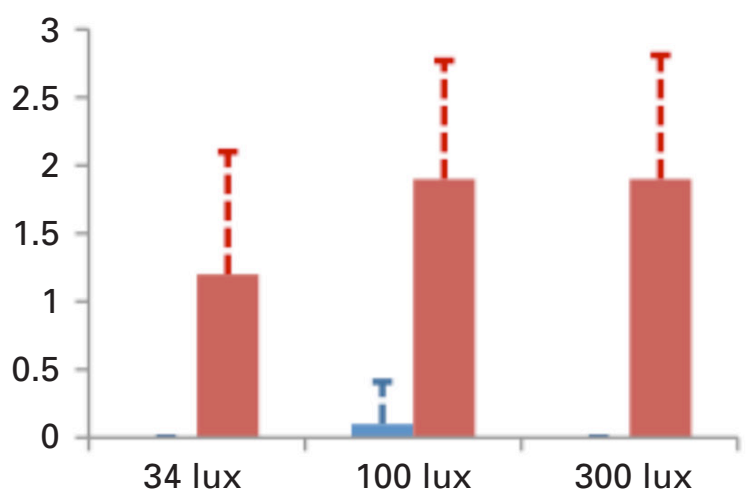

Preterm infants at 32 weeks PMA

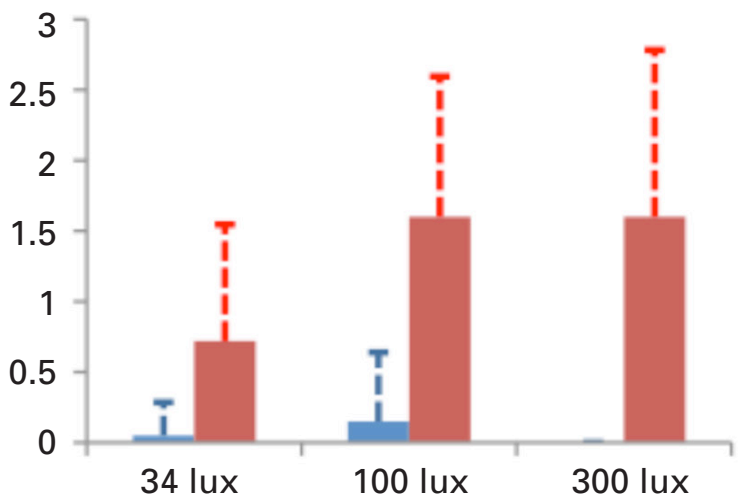

Preterm infants at 40 weeks PMA

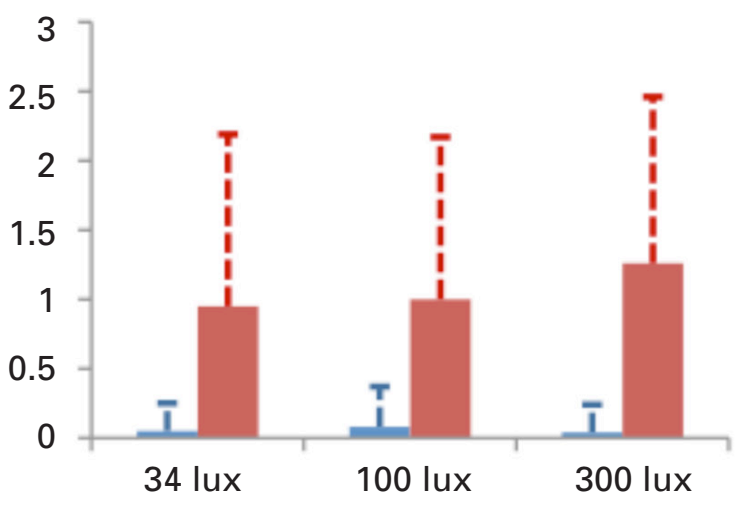

Preterm infants at 36 weeks PMA

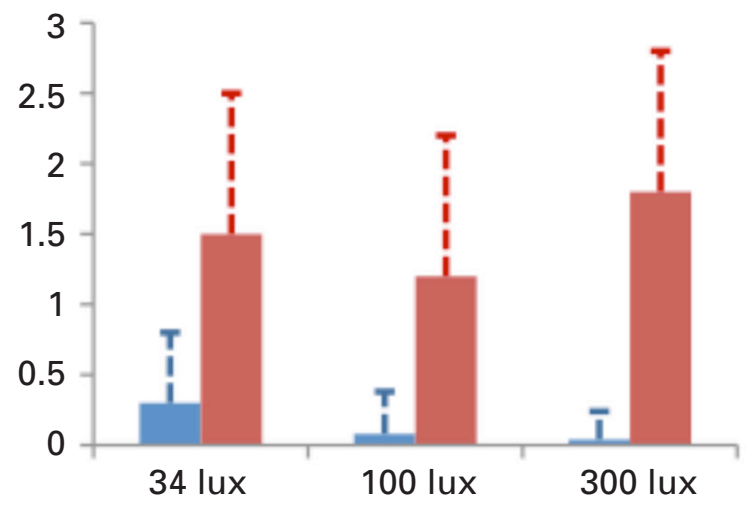

Term newborn infants

Mean NFCS scores during pre-stimulation periods are represented in blue and during post-stimulation periods in red.

The vertical bars represent the standard deviation. ${ }^{*} p<0.05$, Student's $T$ test 